\title{
Variations in Pulp/Tooth Area Ratio as an Indicator of Age: a Preliminary Study*
}

\begin{abstract}
This paper details a method for age determination of adults from single rooted teeth. The sample consisted of 100 Italian white Caucasian patients ( 46 men, 54 women) aged between 18 and 72 years. The single rooted maxillary right canine was utilized in this preliminary study. Pulp/root ration, tooth length, pulp/tooth length ratio, pulp/tooth area and pulp/root width ratios at three different levels were computed. Pearson's correlation coefficients between age and these variables showed that the ratio between pulp and tooth area correlated best with age $\left(r^{2}=0.85\right)$. Stepwise multiple regression models yielded a linear relationship between pulp/root width at mid-root level and chronological age and a linear relationship when pulp/tooth area was compared to age. Statistical analysis indicated that these two variables explain $84.9 \%$ of variations in estimated chronological age. The median of the absolute value of residual errors between actual and estimated ages was less than four years.
\end{abstract}

KEYWORDS: forensic science, age determination by teeth, forensic odontology, dental pulp, pulp/tooth area, stepwise linear regression

Several papers in the field of forensic odontology deal with estimating chronological age in humans (1-8). Although skeletal radiology is the most widespread method for age estimation in living subjects, new techniques have allowed great improvements in the X-ray processing of teeth. These techniques are better suited for age estimations than bones in adults $(4,5)$. The most widely used methods of age estimation depend upon subjective assessment of tooth development. Inspection of radiographs (by magnifying glass and caliper) and subsequent comparisons with radiographic images, drawings and descriptions in charts yield "maturity scores" $(1-3,7,8)$.

Alternative approaches based on digitalization of panoramic radiographs and their computerized storage have recently become available (6). These procedures exploit image analysis to obtain nondestructive metric measurements of both pulp chambers and teeth. Forensic odontologists may now utilize these techniques that are relatively precise and accurate (6). Computer-assisted image analysis avoids the bias inherent in observer subjectivity, improves the reliability and the statistical analysis of data.

A number of statistical methods have been proposed for age determination of adults. Most of these methods are based on establishing correlations between individual age (dependent variable) and some significant tooth parameters (predictors) obtained from digitized radiographs (9). The most frequently used statistical technique for age estimation in forensic dentistry is multiple linear regression, although partial least-squares linear regression, polynomial regression, and other robust regression methods have also been applied.

\footnotetext{
${ }^{1}$ Institute of Forensic Medicine, University of Macerata, Macerata, Italy.

${ }^{2}$ Institute of Biochemical Biotechnologies, Faculty of Medicine, Polytechnical University of Marches, Ancona, Italy.

* This work was funded by a grant to the Polytechnical University of Marches, MURST.

Received 8 Oct. 2003; and in revised form 16 Nov. 2003; accepted 16 Nov. 2003; published Xxxx.
}

The purpose of the present investigation is to present a method for assessing chronological age based on the relationship between age and measurement of the pulp/tooth area ratio (AR) on single-rooted teeth. In an attempt to improve the precision and reliability of age estimations, other dental features were also taken into account and the different statistical models were compared.

\section{Materials and Methods}

\section{Subjects and Materials}

Orthopantomographs of 100 Italian white Caucasian patients (46 men, 54 women) aged between 18 and 72 years were analyzed (Table 1). X-rays were digitized using a scanner and images were recorded in a computer file. Image analysis showed how canines, incisors and second premolars yield the clearest digital images. For this preliminary study, the right maxillary canine was selected, because it was always present and was the most suitable for measurement.

Radiographic images of canines (RIC) were processed using a computer-aided drafting program (AutoCAD2000, Install Shield $3.0,1997)$. Twenty points from each tooth outline and ten points for each pulp outline were identified and used to evaluate tooth and pulp areas. Measurements of the RIC yielded pulp and tooth areas; tooth, pulp and root lengths; and pulp and root widths, at three different levels. All RIC measurements were carried out by the same observer. To test intra-observer reproducibility, a random sample of twenty RIC were re-examined after an interval of two weeks.

\section{Statistical Analyses}

The following morphological variables were recorded (9): $p=$ pulp/root length; $r=$ pulp/tooth length; $a=$ pulp/root width at enamel-cementum junction (ECJ) level; $\mathrm{c}=$ pulp/root width at midroot level; $b=$ pulp/root width at midpoint level between ECJ level 
TABLE 1-Age and gender distribution of study sample.

\begin{tabular}{lccc}
\hline $\begin{array}{c}\text { Age } \\
\text { (Years) }\end{array}$ & $\begin{array}{c}\text { No. of } \\
\text { Males }\end{array}$ & $\begin{array}{c}\text { No. of } \\
\text { Females }\end{array}$ & Total \\
\hline $18-29$ & 11 & 12 & 23 \\
$30-39$ & 15 & 15 & 30 \\
$40-49$ & 9 & 15 & 24 \\
$50-59$ & 6 & 5 & 11 \\
$60-69$ & 5 & 5 & 10 \\
$70-73$ & 0 & 2 & 2 \\
Total & 46 & 54 & 100 \\
\hline
\end{tabular}

and mid-root level; $\mathrm{AR}=$ pulp/tooth area ratio. These measurements were normalized to allow for angulation distortion (9).

The morphological variables, the age and the subjects' gender were entered in a Microsoft EXCEL ${ }^{\circledR}$ spreadsheet for use as predictive variables for age estimation. Unlike other methods (9), the $\mathrm{t}$ ratio (tooth/root length) in measured morphologic variables, because it is functionally dependent on $\mathrm{p}$ and $\mathrm{r}(t=\mathrm{p} / \mathrm{r})$ and therefore does not add information concerning dental age. The chronological age was calculated by subtracting date of birth from the date of radiograph. Intra-observer reproducibility of measurements was studied using a paired $t$-test.

Correlation coefficients were evaluated between age and predictive variables. A multiple linear regression model for age prediction was developed by selecting those variables, which contributed significantly to age estimations using the stepwise selection method. Analysis of covariance (ANCOVA) was then applied to study possible interactions between significant morphological variables and gender. Statistical analysis was performed with S-PLUS 6 statistical programs (S-PLUS ${ }^{\circledR} 6.1$ for Windows PROFESSIONAL EDITION Release 1). The significance threshold was set at $5 \%$.

\section{Results}

There were no statistically significant intra-observer differences between the paired sets of measurements carried out on the RIC $(p=0.64)$.

Pearson's correlation coefficients between age and morphological variables (Table 2) showed that the ratios between length measurements correlated worst with age. In particular, the variable $\mathrm{p}$ (ratio between pulp and root length) was not significantly correlated with age and was therefore excluded from further statistical analysis.

All other correlation coefficients between age and morphological variables were significant and negative. The subjects' age was modeled as a linear function of the morphological variables (predictors). A stepwise regression procedure was applied to optimize the model. The results (Table 3 ) show that only variables AR and c contributed significantly to the fit. The regression model utilizing $\mathrm{AR}$ and $\mathrm{c}$, yielding the following linear regression formula:

$$
\text { Age }=86.53-457.15 \quad \text { AR }-22.98 \quad c
$$

This model had the lowest AIC (Akaike Information Criterion) value (624.0) among the considered linear models. There was a modest improvement in model fit when all five predictors were used. The full model explained $85.1 \%$ of total variance, whereas the model, with the AR and c variables explained $84.9 \%\left(r^{2}=0.849\right)$.

The median of the absolute values of residuals (observed age minus predicted age) was 3.7 years, with a quartile deviation of 4.3 years.
TABLE 2-Correlation coefficients between age and predictive morphological variables.

\begin{tabular}{|c|c|c|c|c|c|}
\hline RA & $\mathrm{p}$ & $\mathrm{r}$ & $\mathrm{a}$ & $\mathrm{b}$ & $\mathrm{c}$ \\
\hline$-0,92$ & $0,11^{*}$ & $-0,21 \dagger$ & $-0,31$ & $-0,38$ & $-0,42$ \\
\hline \multicolumn{6}{|c|}{$\begin{array}{l}{ }^{*} \text { No significance } p<0.05 \\
\dagger p=0.04\end{array}$} \\
\hline \multicolumn{6}{|c|}{$\begin{array}{l}\text { TABLE 3-Stepwise regression analysis predicting chronological age } \\
\text { from selected predictors. }\end{array}$} \\
\hline & \multicolumn{2}{|c|}{$\begin{array}{l}\text { Regression } \\
\text { Coefficient }\end{array}$} & $\begin{array}{l}\text { Standard } \\
\text { Error }\end{array}$ & $\mathrm{t}_{97 ; 0.05}$ & $p$-level \\
\hline Intercept & \multicolumn{2}{|c|}{86.53} & 2.26 & 38.26 & $<0.001$ \\
\hline RA & \multicolumn{2}{|c|}{-457.15} & 21.92 & -20.86 & $<0.001$ \\
\hline $\mathrm{c}$ & \multicolumn{2}{|c|}{-22.98} & 11.17 & -2.06 & 0.042 \\
\hline
\end{tabular}

TABLE 4-Summary table of ANCOVA.

\begin{tabular}{lrrrrr}
\hline & df & \multicolumn{1}{c}{ SS } & \multicolumn{1}{c}{ MS } & \multicolumn{1}{c}{ F } & \multicolumn{1}{c}{$\mathrm{p}$} \\
\hline Intercept & 1 & 41219.17 & 41219.17 & 1433.59 & $<0.001$ \\
RA & 1 & 12439.44 & 12439.44 & 432.64 & $<0.001$ \\
c & 1 & 128.55 & 128.55 & 4.47 & 0.037 \\
Gender & 1 & 11.67 & 11.67 & 0.41 & 0.526 \\
Error & 96 & 2760.23 & 28.75 & & \\
Total & 99 & 18374.91 & & & \\
\hline
\end{tabular}

The residual plot (Fig. 1, left panel) shows no obvious pattern and only three observations appeared to be outside the expected boundaries. The observed versus predicted plot (Fig. 1, right panel) shows that the regression model fits the trend of the data reasonably well. Hence, both diagnostic plots support the chosen model. Only a small increase in the residual could be seen when the simplest linear model with only AR as covariate was used (age $=84.31-473.86$ AR). It should also be noted that, although it was not the best fit, this simple linear model explained $84.26 \%$ of total variance.

To study the possible effect of gender on the linear regression model, an analysis of covariance was performed (ANCOVA). According to the previous analysis, covariates AR and $\mathrm{c}$ had a significant influence on the age of subjects (Table 4). The small F-value which accounts for gender $(F=0.406 ; p=0.526)$ revealed that gender does not significantly influence the regression model used to estimate chronological age for both female and male groups.

\section{Discussion}

The need to estimate the age of living individuals is becoming increasingly important in forensic odontology. There are increasing numbers of immigrants (illegal or otherwise) who arrive in a country without acceptable identification papers and, more generally, individuals with missing or uncertain birth data. The study of the morphological parameters of teeth on dental X-rays of adult humans is more reliable than most other methods for age estimation.

Several studies show that morphological measurements can be reliably utilizing panoramic dental radiographs. These studies require that some corrections are made to take into account individual variability of tooth size, differences in magnification of radiographs and angulation between X-ray beam and film. Some studies $(9,10)$ suggest using the ratio between pulp area and tooth, and the ratios of length and width of the pulp/tooth area, instead of their direct measurement. When area, length and width of pulp and tooth were 

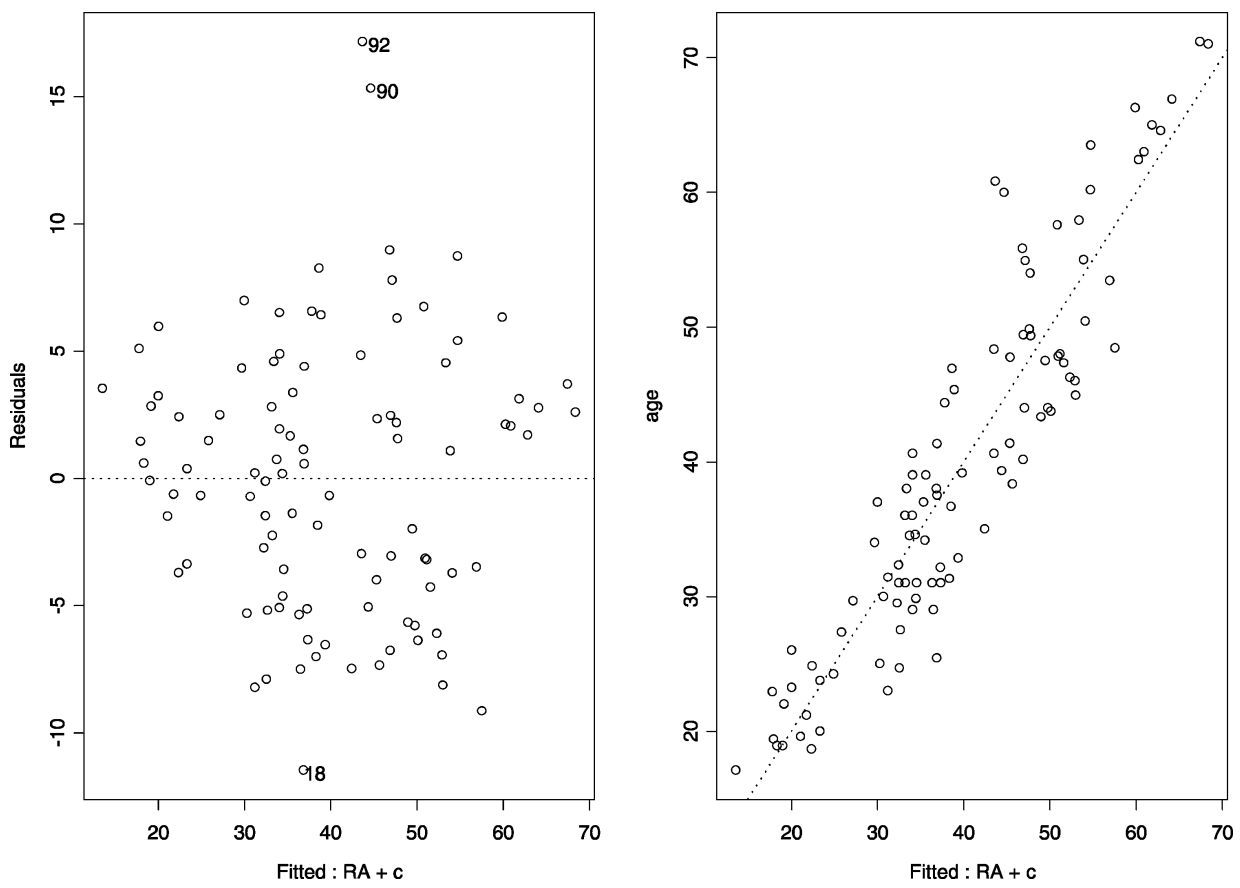

FIG. 1-Plots of residuals against fitted values (left panel) and of observed versus predicted values (right panel), with regression model 1.

measured on digitized images of canines, the paired t-test showed that there were no significant intra-observer differences. It can be concluded that the RIC technique can produce reliable and reproducible intra-observer measurements.

In the sample of 100 individuals, the $\mathrm{AR}$ (pulp/tooth area ratio) of the right maxillary canine proved to be closely correlated with subjects' chronological age. The application of the RIC method for age estimation seems promising $(10,11)$. Further study will include applying the RIC method to other single rooted teeth such as incisors and second premolars.

This preliminary study was limited to the right maxillary canine because it was always present and generally showed clearer images than other single rooted teeth. Nevertheless, including other single rooted teeth and their morphological parameters should provide a better estimate of chronological age. Statistical analysis on regression models shows that the RA is better correlated with chronological age than the linear measurement ratio, and the pulp/root ratio. Width is a better indicator of age than length.

The RIC method yields a median of the absolute value of residual error of less than 4 years, and a standard error of estimate of 5.35 years. It is therefore comparable to other age estimation methods in adults. This method is reasonably accurate considering that only one canine was evaluated for each subject. Analysis of covariance showed that gender had no significant influence on age estimation when only right maxillary canine measurements were utilized. Gender was not included as a factor in the model equation. Repetitive measurements must be carried out by other independent observers in order to verify inter-observer reproducibility (12). Also the determination of minority/majority age will be an important field of future application.

In the future, image analysis programs which can recognize pulp outlines in a radiographic images will be very useful in minimizing human manual measurement of morphological parameters, and will probably reduce both inter- and intra-observer variability.

Other planned studies include attempts to reduce the residual standard error and a comparison of multiple linear regression models for age estimation with other statistical methods such as partial least-squares, robust regression methods, or other non-linear regression procedures.

\section{References}

1. Demirjian A, Goldstain H, Tanner JM. A new system of dental age assessment. Hum Biol 1973;45:221-7.

2. Demirjian A, Goldstain H. New systems for dental maturity based on seven and four teeth. Ann Hum Biol 1976;3:411-21.

3. Mincer HH, Harris EF, Berryman HE. The A.B.F.O. study of third molar development and its use as an estimator of chronological age. J Forensic Sci 1993;38:379-90.

4. Liversidge HM, Herdeg B, Rösing FW. Dental age estimation of non-adults: a review of methods and principals. In: Alt KW, Rösing FW, Teschler-Nicola M, editors. Dental anthropology. Fundamentals, limits and prospects. Vienna: Springer, 1998;420-42.

5. Rösing FW, Kvaal SI. Dental age in adults: a review of estimation methods. In: Alt KW, Rösing FW, Teschler-Nicola M, editors. Dental anthropology. Fundamentals, limits and prospects. Vienna: Springer, 1998;443-68.

6. Willems G. A review of the most commonly used dental age estimation techniques. J Forensic Odontostomatol 2001;19:9-17.

7. Teivens A, Mörnstad H. A modification of the Demirjian method for age estimation in children. J Forensic Odontostomatol 2001;19:26-30.

8. Mesotten K, Gunst K, Carbonez A, Willems G. Dental age estimation and third molars: a preliminary study. Forensic Sci Int 2002;129:110-5.

9. Kvaal SI, Kolveit KM, Thomsen IO, Solheim T. Age estimation of adults from dental radiographs. Forensic Sci Int 1995;74:175-85.

10. Kvaal SI, Solheim T. A non-destructive method for age estimation. J Forensic Odontostomatol 1994;12:6-11.

11. Kolltveit KM, Solheim T, Kvaal SI. Methods of measuring morphological parameters in dental radiographs. Comparison between image analysis and manual measurements. Forensic Sci Int 1998;94:87-95.

12. Willems G, Moulin-Romsee C, Solheim T. Non-destructive dental-age calculation methods in adults: intra- and inter-observer effects. Forensic Sci Int 2002;126:221-6.

Additional information and reprint requests:

Roberto Cameriere, M.D.

Institute of Forensic Medicine-University of Macerata

Via Don Minzoni n. 9

62100 Macerata (Italy)

E-mail: rmaigret@libero.it 